\title{
Ballistic versus diffusive magnetoresistance of a magnetic point contact
}

\author{
L.R. Tagirov ${ }^{1}$, B.P. Vodopyanov ${ }^{2}$, and K.B. Efetov $^{3,4}$ \\ ${ }^{1}$ Kazan State University, Kazan 420008, Russia \\ ${ }^{2}$ Kazan Physico-Technical Institute of RAS, Kazan 420029, Russia \\ ${ }^{3}$ Theoretische Physik III, Ruhr-Universität Bochum, 44780 Bochum, Germany \\ ${ }^{4}$ L.D. Landau Institute for Theoretical Physics, Moscow, Russia
}

(November 3, 2018)

The quasiclassical theory of a nanosize point contacts (PC) between two ferromagnets is developed. The maximum available magnetoresistance values in PC are calculated for ballistic versus diffusive transport through the area of a contact. In the ballistic regime the magnetoresistance in excess of few hundreds percents is obtained for the iron-group ferromagnets. The necessary conditions for realization of so large magnetoresistance in PC, and the experimental results by García et al are discussed.

PACS numbers: 74.80.Dm, 74.50.+r, 74.62.-c

In recent experiments on study of $\mathrm{Ni}-\mathrm{Ni}$ and $\mathrm{Co}-\mathrm{Co}$ point contacts (PC), a surprisingly high negative mage netoresistance exceeding $200 \%$ has been discovered 1 , The set up of the experiment was typical for observation of giant magnetoresistance (GMR), the effect observed earlier in hybrid systems involving ferromagnetic and normal multilayer metals. 3 - However, for the multilayer structures the typical change of the resistance reached $10 \%-30 \%$, which is considerably lower than the corresponding values of Refs. 1,2.2. So, one can come easily to the conclusion that the main contribution to the MR comes from the region of the PC.

A negative magnetoresistance can be due to scattering on domain walls (DW) and this effect has been considered in a number of works 1 giving typical values of MR in a range of few percents. Such considerably low values of the MR were obtained assuming that realistic widths of the DW were large, which resulted in a low scattering amplitudes. Considering sharp $\mathrm{DW}$ in the ballistic regime one comes to values $\sim 70 \%$.

The fact that a sharp DW may give large MR was used in Ref. 2 to explain the anomalously large values of MR in the experiments on the point contacts.1. However, the theory in Ref. 2 is the perturbation theory, it can not be applied to the explanation of $300 \%$ effect. The diminishing of the width of DW, when decreasing the size of the constriction was demonstrated by Bruno. The DW width becomes comparable with PC length, and magnetization rotates almost abruptly inside the constriction. This conclusion holds until the diameter of $\mathrm{PC}$ is smaller than its actual length. With further increase of constriction size (diameter) the wall will bend outside of PC, and simple energy considerations show that the DW width will be of the order of PC size.10

The regime of conductance quantization in magnetic
PC has been considered by Imamura et al 11 They obtained that, if the spin of conduction electron cannot rotate in DW pinned to the constriction, then magnetoresistance acquires oscillations as a function of $\mathrm{PC}$ size with amplitude exceeding $1000 \%$.

In this paper we develop a quasiclassical theory of electric transport through magnetic PC taking into account scattering by impurities, thus covering the ballistic $(l>a)$ and diffusive $(l<a)$ regimes $(l$ is the mean free path and $a$ is the radius of the contact). The typical PC size, which is beyond the quantization regime, $2 a \geq 8 \AA$, may be well described within the quasiclassical (QC) approximation $\left(2 a \gg \lambda_{F}=2 \pi / p_{F} \sim 6 \AA, \lambda_{F}\right.$ and $p_{F}$ are the Fermi wavelength and momentum).

We believe that extremely large magnetoresistance can be obtained, if the strong reflection of spin-polarized current carriers on the $\mathrm{PC}$ area is achieved at antiparallel (AP) alignment of magnetizations in contacting ferromagnets. This is realized, if there is mismatch in the spinsubbands Fermi-momenta of contacting magnets. For AP alignment $p_{F 1 \uparrow}=p_{F 2 \downarrow}$, and $p_{F 1 \downarrow}=p_{F 2 \uparrow}$. Let us assume that $p_{F 1 \uparrow} \gg p_{F 1 \downarrow}$. Then subband with the smaller value of the Fermi momenta, which is minority subband, can not accept momenta transferred from the opposite side of the PC, which is majority subband with the same spin projection. As a result, only a narrow incidence angles cone around the normal to the interface is responsible for the charge transport across the PC. Electrons with more inclined trajectories are completely reflected. Thus, the partial transmission at the steep incidence, and the total reflection at slanting incidence provide high boundary resistance of $\mathrm{PC}$.

The necessary condition for realization of the above scenario is the conservation of electron spin orientation when crossing the domain wall. The orientation conserves, if the DW width $d_{w}$ is shorter than the length $d_{s}$, at which the electron spin quantization axis adjusts the varying direction of local exchange field. For ballistic transmission through $\mathrm{PC} d_{s}=v_{F} T_{1}$, where $T_{1}$ is the longitudinal relaxation time of conduction electron magnetization - the Overhauser time.12 At this condition the transmission process looks like transmission through abrupt DW, and the description of the electron transport through PC with boundary conditions at PC interface is valid.

The PC model we consider is the circular hole of the radius $a$ made in a membrane, which divides the space 
on two half-spaces, occupied by single-domain ferromagnetic metals. The membrane is impenetrable for the quasiparticles carrying a current, however the thickness of the membrane in the model is assumed to be vanishing. The $z$-axis of coordinate system is chosen perpendicular to the membrane plane. The electron motion on both sides of the contact can be described by the equations for quasiclassical (QC) Green functions derived by Zaitsev.13 They are in fact the Boltzmann equations in the $\tau$-approximation:

$$
\begin{array}{r}
v_{z} \frac{\partial g_{a}}{\partial z}+\mathbf{v}_{\|} \frac{\partial g_{s}}{\partial \vec{\rho}}+\frac{1}{\tau}\left(g_{s}-\bar{g}_{s}\right)=0 \\
v_{z} \frac{\partial g_{s}}{\partial z}+\mathbf{v}_{\|} \frac{\partial g_{a}}{\partial \vec{\rho}}+\frac{g_{a}}{\tau}=0
\end{array}
$$

$g_{s}$ and $g_{a}$ are symmetric and antisymmetric with respect to $z$ - projection of quasiparticle momentum QC GF (Green functions integrated over the energy variable), $\mathbf{v}$ is the vector of the Fermi velocity, $v_{z}=v_{F} \cos \theta$, $v_{\|}^{2}=v_{F}^{2}-v_{z}^{2}$, angle $\theta$ is measured from the $z$-axis, $v_{F}$ is the modulus of $\mathbf{v}$, the bar over $\bar{g}_{c}$ means the averaging over the solid angle. We assume that the spin-mixing process is weak, therefore we consider spin channels as independent and omit the spin-channel indices in (11) and expressions below.

The boundary conditions to equation (11) for the specular scattering ( $p_{E 1} \alpha \sin \theta_{1}=p_{F 2 \alpha} \sin \theta_{2} \equiv p_{\|}$) at the interface $z=0$ are:13

$$
\begin{gathered}
g_{a 1}(0)=g_{a 2}(0)=\left\{\begin{array}{c}
g_{a}(0), \quad p_{\|}<p_{F 1}, p_{F 2} \\
0, \quad \min \left(p_{F 1}, p_{F 2}\right)<p_{\|}
\end{array},\right. \\
2 R g_{a}(0)=-D\left(g_{s 2}-g_{s 1}\right),
\end{gathered}
$$

where subscript 1 or 2 labels left- or right-hand side of the contact, respectively, $p_{F i}$ is the Fermi momentum of $i$-th side, $p_{\|}$is the projection of the Fermi momentum vector on the PC plane. $D$ and $R=1-D$ are the exact quantum mechanical transmission and reflection coefficients that can be considered either as phenomenological parameters or calculated for models of interest. The second line in the first boundary condition in (2) explicitly quantifies the total reflection for inclined trajectories, described qualitatively above.

The density of a current through the contact may be written as

$$
j^{z}(z, \vec{\rho}, t)=-\frac{e p_{F \min }^{2}}{2 \pi} \int_{0}^{\pi / 2} d \Omega_{\theta} \cos \theta g_{a}(z, \vec{\rho}, t) .
$$

The total current through the area of the contact is

$$
I^{z}(z \rightarrow 0, t)=a \int_{0}^{\infty} d k J_{1}(k a) j^{z}(0, k, t) .
$$

In the above equations $p_{F \min }=\min \left(p_{F 1}, p_{F 2}\right), J_{1}(x)$ is the Bessel functions, $j^{z}(0, k, t)$ is the Fourier-transform of current density, Eq. (3), over the in-plane coordinate $\rho$. The cylindrical symmetry of the problem has been used upon derivation of Eq. (4).

We search a solution for $g_{s}$ in the form $\left(k_{B}=\hbar=1\right)$ :

$$
g_{s}(\varepsilon)=\tanh \frac{\varepsilon}{2 T}+f_{s}(\varepsilon)
$$

where the first term is the equilibrium value of $g_{s}$ in the leads far away of PC. Substitution of (5) into (11) and Fourier transformation over the variable $\rho$ leads to equations, the exact solution of which reads

$$
f_{s}(z)=g_{a}(z) \operatorname{sgn}(z)+\frac{1}{l_{z}} \int_{-\infty}^{\infty} d \xi e^{-\varkappa|\xi-z|} \bar{f}_{s}(\xi, k)
$$

where

$$
\varkappa=\frac{1-i \mathbf{k} \mathbf{l}_{\|}}{l_{z}}
$$

$l=\tau v_{F}$ is the mean free path, $l_{z}=l \cos \theta, l_{\|}^{2}=l^{2}-l_{z}^{2}$. Integrating Eq. (6) over the solid angle we obtain

$$
\bar{f}_{s}(z>0)=\bar{g}_{a}+\int_{z}^{\infty} d \xi K(\xi-z) \bar{f}_{s}(\xi, k),
$$

where the kernel $K(\eta)$ is $(x=\cos \theta)$

$$
K(\eta)=\frac{1}{l} \int_{0}^{1} d x \frac{e^{-\frac{\eta}{l x}}}{x} J_{0}\left(k \eta \frac{\sqrt{1-x^{2}}}{x}\right) .
$$

If the mean free path $l$ is short $(l \ll a)$, the second term in Eqs. (6) and (8) dominates and the integrand of Eq. (8) is the product of rapidly decreasing on the distance $l$ kernel $K(\eta)$ and slowly decreasing function $\bar{f}_{s}$. That is why we may take out $\bar{f}_{c}(k, \xi)$ from the integral (\&) at the point $\xi=z$. Within this approximation we obtain

$$
\bar{f}_{s}(z, k)=\bar{g}_{a}(z, k)(1-\lambda(k))^{-1}
$$

where

$$
\lambda(k)=\int_{0}^{\infty} d \xi K(\xi-z)=\frac{1}{k l} \arctan k l .
$$

Substituting Eq. (10) into Eq. (6), and using the boundary conditions (2) we obtain the equation for the antisymmetric combination $g_{a}$ :

$$
\begin{aligned}
g_{a}(0, k)=-\frac{1}{2} D( & \left.\tanh \frac{\varepsilon}{2 T}-\tanh \frac{\varepsilon-e V}{2 T}\right) \gamma_{k} \\
& -\frac{D}{1-\lambda_{1}} \frac{1}{2 l_{z 1}} \int_{-\infty}^{0} d \xi e^{\varkappa_{1} \xi} \bar{g}_{a 1}(\xi) \\
& -\frac{D}{1-\lambda_{2}} \frac{1}{2 l_{z 2}} \int_{0}^{\infty} d \xi e^{-\varkappa_{2} \xi} \bar{g}_{a 2}(\xi),
\end{aligned}
$$


where

$$
\gamma_{k}=\int_{0}^{a} \rho d \rho \int_{0}^{2 \pi} e^{i \mathbf{k} \vec{\rho}} d \varphi=\frac{2 \pi a}{k} J_{1}(k a),
$$

$V$ is the bias voltage.

To find $g_{a}(0, k)$ we average Eq. (12) over the solid angle, exploit the continuity of $g_{a}$ at the interface, Eq. (2), and, again, use the fact that in the limit $l \ll a$ the kernel in the integral over $x$ in the second and third terms of averaged Eq. (12) is a function, rapidly decreasing at distance $l$. Of course, in the ballistic regime $(l>$ a ) this approximation is no longer valid, but, in this regime the first (exact) term in Eq. (12) dominates the approximate terms with integrals. So, in the ballistic limit the approximation done does not bring a big error either. Although the approximation may not be valid in the intermediate regime, the suggested scheme can be used as an interpolation.

Now we find easily $\bar{g}_{a}(0, k)$, make consecutive substitutions into (12), (3), (4), and, finally, obtain the general expression for the current through PC:

$$
I^{z}=\frac{e^{2} p_{F \min }^{2} a^{2} V}{2 \pi} \int_{0}^{\infty} \frac{d k}{k} J_{1}^{2}(k a)\langle D F(k, \theta) \cos \theta\rangle,
$$

where

$$
\begin{gathered}
F(k, \theta)=1-\left[\frac{1}{2\left(1-\lambda_{1}\right) \varkappa_{1} l_{z 1}}+\frac{1}{2\left(1-\lambda_{2}\right) \varkappa_{2} l_{z 2}}\right] \\
\times \frac{\tilde{\lambda}_{1}}{1+\frac{\tilde{\lambda}_{1}}{2\left(1-\lambda_{1}\right)}+\frac{\tilde{\lambda}_{2}}{2\left(1-\lambda_{2}\right)}} \\
\tilde{\lambda}_{i}=\left\langle\frac{D}{\varkappa_{i} l_{z i}}\right\rangle=\int_{0}^{1} d x \frac{D(x)}{\sqrt{1+k^{2} l_{i}^{2}\left(1-x^{2}\right)}},
\end{gathered}
$$

$\langle\ldots\rangle$ means averaging over the solid angle. Eqs. (14) and (15) are the basic analytical result of the paper, which expresses the current in terms of parameters $D$, $l, a, p_{F}$ characterizing the system.

Now we calculate the magnetoresistance of PC between two identical ferromagnets. It can be expressed via the conductances $\sigma=I / V$ as follows

$$
M R=\frac{R^{A P}-R^{P}}{R^{P}}=\frac{\sigma^{P}-\sigma^{A P}}{\sigma^{A P}},
$$

where $R^{P}\left(\sigma^{P}\right)$ stands for the resistance (conductance) at parallel alignment of magnetizations of contacting ferromagnets, and $R^{A P}\left(\sigma^{A P}\right)$ is for the antiparallel alignment of magnetizations. For the parallel alignment the net current is the sum of currents for both (independent) spin channels, $D=1, \widetilde{\lambda}_{i}=\lambda_{i}$. Labelling the quantities by arrow-up/down notations we write down

$$
\begin{array}{r}
\sigma^{P}=\sigma_{\uparrow \uparrow}^{z}+\sigma_{\downarrow \downarrow}^{z}=\frac{e^{2}\left(p_{F \uparrow}^{2}+p_{F \downarrow}^{2}\right)\left(\pi a^{2}\right)}{4 \pi^{2}} \int_{0}^{\infty} \frac{d k}{k} J_{1}^{2}(k a) \\
\quad \times\left\{\frac{p_{F \uparrow}^{2}}{p_{F \uparrow}^{2}+p_{F \downarrow}^{2}} \frac{k^{2} l_{\uparrow}^{2}}{\left(1+\sqrt{1+k^{2} l_{\uparrow}^{2}}\right)^{2}}+(\uparrow \rightleftarrows \downarrow)\right\} .
\end{array}
$$

The prefactor in Eq. (18) is nothing but the sum of Sharvin 14 conductances for the spin channels. For the $\mathrm{AP}$ alignment of magnetizations the conductance is

$$
\begin{gathered}
\sigma^{A P}=\frac{e^{2} p_{F \downarrow}^{2}\left(\pi a^{2}\right)}{\pi^{2}} \int_{0}^{\infty} \frac{d k}{k} J_{1}^{2}(k a) \int_{0}^{1} d x x(D(x))_{\uparrow \downarrow} \\
\times\left\{1-\left[\frac{1-\lambda^{\uparrow}}{\sqrt{1+k^{2} l_{\uparrow}^{2}\left(1-x^{2}\right)}}+\frac{1-\lambda^{\downarrow}}{\sqrt{1+k^{2} l_{\downarrow}^{2}\left(1-x^{2}\right)}}\right]\right. \\
\left.\times \frac{(\bar{D})_{\uparrow \downarrow}}{2\left(1-\lambda^{\uparrow}\right)\left(1-\lambda^{\downarrow}\right)+\widetilde{\lambda}_{\uparrow \downarrow}^{\uparrow}\left(1-\lambda^{\downarrow}\right)+\widetilde{\lambda}_{\uparrow \downarrow}^{\downarrow}\left(1-\lambda^{\uparrow}\right)}\right\},
\end{gathered}
$$

where $(D(x))_{\uparrow \downarrow}$ stands for the transmission coefficient of the interface at AP alignment. For the mechanism of magnetoresistance discussed above, $(D(x))_{\uparrow \downarrow}$ can be found from the solution of Schrödinger equation for the particle moving in the step-like potential landscape 15

$$
(D(x))_{\uparrow \downarrow}=\frac{4\left(v_{z 1}^{\uparrow}\right)_{\uparrow}\left(v_{z 2}^{\uparrow}\right)_{\downarrow}}{\left(\left(v_{z 1}^{\uparrow}\right)_{\uparrow}+\left(v_{z 2}^{\uparrow}\right)_{\downarrow}\right)^{2}}=(D(x))_{\downarrow \uparrow}
$$

with $v_{z 2}^{\uparrow}=v_{z 1}^{\downarrow}$ for the antiparallel alignment. The transmission coefficient (20) gives maximum available magnetoresistance values for a particular parameters choice. Neglecting the difference of the effective masses in the spin-subbands we may write down

$$
(D(x))_{\uparrow \downarrow} \simeq \frac{4 x \sqrt{b^{2}+x^{2}}}{\left(x+\sqrt{b^{2}+x^{2}}\right)^{2}},
$$

where

$$
b^{2}=\frac{1-\delta^{2}}{\delta^{2}}, \quad \delta=\frac{p_{F \downarrow}}{p_{F \uparrow}}=\frac{v_{F \downarrow}}{v_{F \uparrow}} \leq 1 .
$$

For the purely ballistic transport $\left(a / l_{\uparrow} \rightarrow 0\right.$, where $l_{\uparrow}$ $\left(l_{\downarrow}\right)$ is the majority (minority) electrons mean free path) all integrals in Eqs. (18), (19) are evaluated analytically, and magnetoresistance reads

$$
M R=\frac{(1-\delta)\left\{5 \delta^{3}+15 \delta^{2}+9 \delta+3\right\}}{8 \delta^{3}(\delta+2)} .
$$

If $\delta=1$, then $M R=0$, i.e. the magnetoresistance vanishes. For the set of $\delta$ values we obtain from (23): 
$\delta=0.5, M R=238 \% ; \delta=0.4, M R=455 \% ; \delta=0.33$, $M R=780 \% ; \delta=0.3, M R=1012 \%$.

In general case the angular integrals in (16) and (19) can be still evaluated analytically, whereas the integrations over $k$ can be done only numerically. The results for the magnetoresistance (17) as function of the contact radius are shown on Fig. 1. The curves show the maximum available MR, that could be realized in $\mathrm{PC}$ with physical parameters displayed on the figure. MR exponentially drops when the size of the contact approaches the mean free path of a material. Then it shows a smooth crossover from ballistic to diffusive regimes of conduction.

Let us discuss the experimental data on magnetoresistance of magnetic PC by García et al. Ni-Ni PC showed maximal $M R \simeq 280 \%$, 1 Co-Co PCshowed maximal $M R \simeq 230 \%$ In the recent paper 16 they quote maximal $M R \simeq 33 \%$ for Fe-Fe PC. To obtain the $M R$ values $280 \%(\mathrm{Ni})$ and $230 \%(\mathrm{Co})$ we have to use the values $\delta(\mathrm{Ni}) \simeq 0.47$ and $\delta(\mathrm{Co}) \simeq 0.5$. These numbers are in the range of the values, obtained experimentally from the single photon threshold photoemission, $\delta(\mathrm{Co}) \simeq$ $0.4,19$ and from ferromagnet/supercondyctor point cop tact spectroscopy: $\delta(\mathrm{Ni}) \simeq 0.59-0.6517 \delta(\mathrm{Ni}) \simeq 0.71 ; 18$ $\delta(\mathrm{Co}) \simeq 0.62-0.65,10(\mathrm{Co}) \simeq 0.68 .18$

If we use the experimental data of Ref. 17 for iron, $\delta(\mathrm{Fe}) \simeq 0.59-0.65$, then in our theory we obtain $M R(\mathrm{Fe})=(100-140) \%$ which is larger than the experimentally measured $33 \% 10$ The justification of our model suggests that observed MR does not solely confined to a value of polarization $\delta$. We believe that the basic condition for observation of upper MR limit, $d_{w} \ll d_{s}$, is not fulfilled in the Fe-Fe PC experiment.16 $T_{1}$ is proportional to the squared magnetic moment and the integral of exchange between conduction electrons and localized moments, and proportional to conduction electrons density of states at Fermi level. All these physical parameters for iron are larger than for cobalt, and especially than for nickel. Therefore we expect, that $T_{1}(\mathrm{Fe})$ at least one order of magnitude shorter than $T_{1}$ for Co and Ni. When $d_{s}(\mathrm{Fe}) \sim d_{w}(\mathrm{Fe})$, the electron spin almost tracks the local exchange field in the domain wall. As a result the reflection of the electrons from DW decreases, and the observed MR does not reach its maximal value.

Let us discuss now the magnetoresistance in the diffusive regime of transport, when the radius of nanohole is much larger than the mean free path $\left(a \gg l_{\uparrow}, l_{\downarrow}\right)$. The giant MR values can be obtained, if the condition of validity of our model, $d_{w} \ll d_{s}$, will be realized in an experiment. In the opposite limit, $d_{w}>d_{s}$, when $\mathrm{PC}$ size is so large that DW becomes smooth and wide, the electron spin will track the local exchange fieldin the domain wall, and MR will level off at Levy-Zhang impurity scattering enhancement mechanism, which can give 2$11 \%$ magnetoresistance. The requirement of abrupt DW with constant width, irrespective of the PC size, can be technologically controlled, if very thin (2-4 monolayers of the thickness $\sim \lambda_{F}$ ) nonmagnetic interlayer is deposited on the PC plane before depositing the second electrode.
Then, just like in CPP transport in multilayers, 3 , the contacting domains will be exchange decoupled, so the magnetization will acquire sudden reversal within the spacer thickness $\sim \lambda_{F}$. In this case our analysis is valid for an arbitrary size of PC.

This work has been supported by Deutsche SFB 491. L.R.T. and B.P.V. acknowledge the support by the Russian Science Foundation through the grant N 00-02-16328 and by CRDF through the grant REC-007. We are grateful to Profs P. Bruno, G.B. Teitelbaum, A.F. Volkov, K.Westerholt and H. Zabel for discussions of the results.

${ }^{1}$ N. García, M. Muñoz, and Y.-W. Zhao, Phys. Rev. Lett. 82, 2923 (1999).

${ }^{2}$ G. Tatara, Y.-W. Zhao, M. Muñoz, and N. García, Phys. Rev. Lett. 83, 2030 (1999).

${ }^{3}$ M.A.M. Gijs, G.E.W. Bauer, Adv. Phys. 46, 285 (1997).

${ }^{4}$ J.-Ph. Ansermet, J. Phys.: Cond. Matt 10, 6027 (1998).

${ }^{5}$ G.G. Cabrera and L.M. Falicov, Phys. Stat. Solidi (b) 61, 539 (1974); 62, 217 (1974).

${ }^{6}$ L. Berger, J. Appl. Phys. 49, 2156 (1978); 69, 1550 (1991).

${ }^{7}$ P.M. Levy, Sh. Zhang, Phys. Rev. Lett. 79, 5110 (1997).

8 J.B.A.N. van Hoof et al, Phys. Rev. B 59, 138 (1999).

${ }^{9}$ P. Bruno, Phys. Rev. Lett. 83, 2425 (1999).

${ }^{10}$ We indebted to Prof. A.F. Volkov who attracted our attention to this point.

${ }^{11}$ H. Imamura, N. Kobayashi, S. Takahashi and S. Maekawa, Phys. Rev. Lett. 84, 1003 (2000).

12 A. Overhauser, Phys. Rev. 89, 689 (1953).

13 A.V. Zaitsev, ZhETF 86, 1742 (1984); Sov. Phys. - JETP 59, 1015 (1984).

14 Yu.V. Sharvin, ZhETF 48, 984 (1965); Sov. Phys. - JETP 21, 655 (1965).

${ }^{15}$ L.D. Landau and E.M. Lifshitz, Quantum Mechanics, §25, Butterworth-Heinemann, Oxford, 1995.

${ }^{16}$ N. García, M. Muñoz and Y.-W. Zhao, Appl. Phys. Lett. 76, 2586 (2000).

${ }^{17}$ R.J. Soulen et al, Science 282, 85 (1998); J. Appl. Phys. 85, 4589 (1999).

18 S.K. Upadhyay, A. Palanisami, R.N. Louie and R.A. Buhrman, Phys. Rev. Lett. 81, 3247 (1998).

19 J.C. Gröbli et al, Physica 204B, 359 (1995).

\section{Figure captions}

Fig.1. The dependence of magnetoresistance on the $\mathrm{PC}$ radius. 